\title{
New nearby white dwarfs from Gaia DR1 TGAS and UCAC5/URAT ^
}

\author{
R.-D. Scholz ${ }^{1}$, H. Meusinger ${ }^{2,3}$, and H. Jahrei $\beta^{4}$ \\ ${ }^{1}$ Leibniz-Institut für Astrophysik Potsdam, An der Sternwarte 16, 14482 Potsdam, Germany \\ e-mail: rdscholz@aip.de \\ 2 Thüringer Landessternwarte Tautenburg, Sternwarte 5, 07778 Tautenburg, Germany \\ e-mail: meus@tls-tautenburg.de \\ ${ }^{3}$ University Leipzig, Faculty of Physics and Geosciences, Linnestr. 5, 04103 Leipzig, Germany \\ ${ }^{4}$ Zentrum für Astronomie der Universität Heidelberg, Astronomisches Rechen-Institut, Mönchhofstraße 12-14, \\ 69120 Heidelberg, Germany \\ e-mail: hartmut@ari.uni-heidelberg.de
}

Received 10 August 2017 / Accepted 28 November 2017

\begin{abstract}
Aims. Using an accurate Tycho-Gaia Astrometric Solution (TGAS) 25 pc sample that is nearly complete for GK stars and selecting common proper motion (CPM) candidates from the 5th United States Naval Observatory CCD Astrograph Catalog (UCAC5), we search for new white dwarf (WD) companions around nearby stars with relatively small proper motions.

Methods. To investigate known CPM systems in TGAS and to select CPM candidates in TGAS+UCAC5, we took into account the expected effect of orbital motion on the proper motion and proper motion catalogue errors. Colour-magnitude diagrams (CMDs) $M_{J} / J-K_{s}$ and $M_{G} / G-J$ were used to verify CPM candidates from UCAC5. Assuming their common distance with a given TGAS star, we searched for candidates that occupied similar regions in the CMDs as the few known nearby WDs (four in TGAS) and WD companions (three in TGAS+UCAC5). The CPM candidates with colours and absolute magnitudes corresponding neither to the main sequence nor to the WD sequence were considered as doubtful or subdwarf candidates.

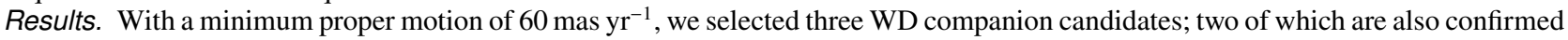
by their significant parallaxes measured in URAT data, whereas the third may also be a chance alignment of a distant halo star with a nearby TGAS star that has an angular separation of about $465 \mathrm{arcsec}$. One additional nearby WD candidate was found from its URAT parallax and $G J K_{s}$ photometry. With HD 166435 B orbiting a well-known G1 star at $\approx 24.6 \mathrm{pc}$ with a projected physical separation of $\approx 700 \mathrm{AU}$, we discovered one of the hottest WDs, classified by us as DA2.0 \pm 0.2 , in the solar neighbourhood. We also found TYC 3980-1081-1 B, a strong cool WD companion candidate around a recently identified new solar neighbour with a TGAS parallax corresponding to a distance of $\approx 8.3 \mathrm{pc}$ and our photometric classification as $\approx \mathrm{M} 2$ dwarf. This raises the question of whether previous assumptions on the completeness of the WD sample to a distance of $13 \mathrm{pc}$ were correct.
\end{abstract}

Key words. astrometry - parallaxes - proper motions - binaries: general - white dwarfs - solar neighborhood

\section{Introduction}

Most of the currently known nearby $(d<25 \mathrm{pc})$ stars were first suspected as solar neighbours because of their high proper motions. The lower limit of high proper motion catalogues has

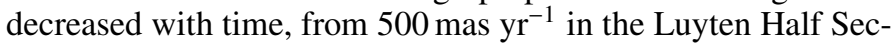

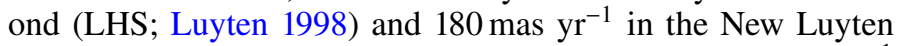
Two Tens (NLTT; Luyten 1995) catalogues, to 150 mas $\mathrm{yr}^{-1}$ in the Lépine and Shara proper motion catalogue of northern stars (LSPM-North; Lépine \& Shara 2005), and now to $40 \mathrm{mas} \mathrm{yr}^{-1}$ in the publicly not yet available SUPERBLINK catalogue (Lépine 2017). Only $15 \%$ of about 3300 stars within 25 pc in the catalogue of nearby stars (CNS; Gliese \& Jahreiß 1995)

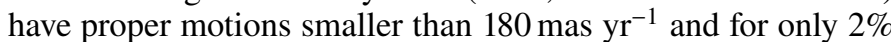

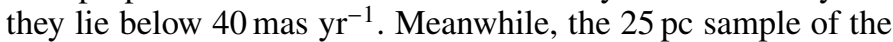
REsearch Consortium On Nearby Stars $\left(\right.$ RECONS $\left.^{1}\right)$ has been

\footnotetext{
^ Partly based on observations with the $2.2 \mathrm{~m}$ telescope of the GermanSpanish Astronomical Centre at Calar Alto, Spain

1 http://www.recons.org/
}

improved with respect to the accuracy of the measured trigonometric parallaxes and contains about 4000 objects in 3000 systems (Henry \& Jao 2015). While the 25 pc census of AFGK-type stars had already been completed by the HIPPARCOS (ESA 1997) mission that surveyed all these bright stars for their trigonometric parallaxes independent of their proper motions and colours, the fainter white dwarfs (WDs) and M-type dwarfs had to be preselected as targets for time-consuming ground-based parallax programmes by their colours and/or proper motions. According to the RECONS, there was an increase of $11 \%$ for WDs and $25 \%$ for $\mathrm{M}$ dwarfs if one considers the immediate solar neighbourhood (the $10 \mathrm{pc}$ sample) in 2012 compared to its status in 2000.

A new unbiased survey that will help to finally complete the stellar, and also partly the substellar, census of the solar neighbourhood independent of proper motion limits is now being carried out by the Gaia mission (Gaia Collaboration et al. 2016b). A first relatively small, still incomplete, and magnitude-limited $(V<11.5)$ subset of very accurate parallaxes was provided with the about two million stars in the Tycho-Gaia Astrometric Solution (TGAS; Lindegren et al. 2016). The TGAS catalogue 
is expected to include the majority of the GK-type stars within $25 \mathrm{pc}$, whereas parallaxes of the brighter AF-type solar neighbours and the fainter nearby WDs and the most frequent solar neighbours, M-type stars, as well as most ML- and some Ttype brown dwarfs (see Smart et al. 2017) will only be provided in later Gaia data releases. The largest intermediate parallax survey between HIPPARCOS and Gaia was provided by Finch \& Zacharias (2016b), who used data from the first U.S. Naval Observatory Robotic Astrometric Telescope Catalog (URAT1; Zacharias et al. 2015). The full URAT Parallax Catalog (UPC; Finch \& Zacharias 2016a) contains more than 112000 stars north of $\delta=-13^{\circ}$ available from Finch \& Zacharias (2016c) with significant parallax measurements, including 53500 stars, for which no previous parallaxes were available. We note that the UPC is not biased towards high proper motion objects.

The positions of all stars published in the first Gaia data release (Gaia DR1; Gaia Collaboration et al. 2016a; Lindegren et al. 2016) were used to improve the proper motion measurements of fainter stars in the 5th United States Naval Observatory CCD Astrograph Catalog (UCAC5; Zacharias et al. 2017). The UCAC5 proper motions are of similar accuracy as those of TGAS (1-2 mas $\mathrm{yr}^{-1}$ ) for stars with $R$ magnitudes between 11 and 15. Out of all 107 million UCAC5 stars, 25 million have proper motion errors smaller than 2 mas $\mathrm{yr}^{-1}$. Therefore, we can search among the typically fainter UCAC5 stars for objects that have a common proper motion (CPM) with a TGAS star. The CPM companions found in UCAC5 can then be assumed to lie at the same distances as their primary stars from TGAS, respectively. The CPM method has usually been applied to high proper motion stars, for which the errors were much smaller than the proper motion values (e.g. Luyten 1997; Li et al. 2014; Lépine 2011). Very wide binaries with projected physical separations of $\gtrsim 1 \mathrm{pc}$ were already analysed in TGAS data alone by Oh et al. (2017) and Andrews et al. (2017). Interestingly, apparent members of open clusters, which lie at distances from the Sun (S cap) between $45 \mathrm{pc}$ and $450 \mathrm{pc}$, were found at very large separations (up to $15 \mathrm{pc}$ ) from their cluster centres (Gaia Collaboration et al. 2017), not only by their CPM status but also according to their common parallaxes in TGAS.

Within the traditional CNS horizon of $25 \mathrm{pc}$ from the Sun, Tremblay et al. (2017) have found and analysed only four WDs directly observed in TGAS. In addition they identified nine such nearby TGAS stars, which have wide WD companions that were too faint to be included in TGAS. Compared to the total number of more than 180 currently known WDs with accurate parallaxes within $25 \mathrm{pc}$ (Subasavage et al. 2017) ${ }^{2}$, the TGAS numbers of directly and indirectly observed WDs are very small. Holberg et al. (2016) have listed 232 WDs with trigonometric parallaxes or spectroscopic distance estimates in their $25 \mathrm{pc}$ sample. Despite the obvious incompleteness of the TGAS $25 \mathrm{pc}$ sample with respect to low-mass stars and WDs, most of which are fainter than the TGAS magnitude limit, and although the UCAC5 does not go deep enough to detect the coolest WDs at about $25 \mathrm{pc}$ either, we aim at a CPM search for unknown nearby WD companions using the very accurate proper motions in both TGAS and UCAC5. In particular, we want to extend the CPM method to those of the known nearest stars that exhibit relatively small proper motions.

\footnotetext{
2 According to http://wwW . denseproject.com/25pc/ more than $20 \%$ of these nearby WDs are components of binary or multiple systems.
}

\section{Common proper motion of nearby wide binaries}

\subsection{The TGAS 25 pc sample}

We have selected 973 stars from TGAS whose parallaxes are larger than 40 mas and cross-identified this TGAS 25 pc sample with the Two Micron All Sky Survey (2MASS; Skrutskie et al. 2006) after converting the TGAS coordinates with epoch 2015 to epoch 2000 taking into account the TGAS proper motions and using TopCat (Taylor 2005). A search radius of 4 arcsec was used as the 2MASS coordinates are given for various epochs between 1997 and 2001. Applying the TGAS proper motions to the 2MASS positions, we then converted the latter to the epoch 2000 as well. The resulting angular distances between the epoch 2000 positions from both catalogues were small (median of 0.12 arcsec, where only a few values are above 0.5 arcsec and a maximum of 1.1 arcsec). All 973 stars were matched and provided with 2MASS $J H K_{s}$ magnitudes (except for one star lacking the $K_{s}$ magnitude). However, only 871 out of the 973 stars have high-quality 2MASS photometry (quality flags "AAA").

The number of TGAS stars within 25 pc corresponds to only a quarter of the already mentioned RECONS 25 pc sample. However, all TGAS stars within $25 \mathrm{pc}$ have trigonometric parallax errors of less than 1 mas (for $\approx 75 \%$ the errors are even $<0.5$ mas), whereas the corresponding RECONS parallaxes were required to have errors of less than 10 mas. Hence the TGAS $25 \mathrm{pc}$ sample is highly incomplete but very accurate with respect to its astrometric measurements. The proper motion errors for TGAS stars within $25 \mathrm{pc}$ ( $\approx 88 \%$ of them are HIPPARCOS stars) are also much smaller than for other TGAS stars (for $\approx 80 \%$ the errors are

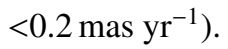

\subsection{Effect of wide orbital motion on proper motions}

When we search for CPM objects to known nearby stars with accurately measured distances, we can estimate the expected influence of orbital motion on the proper motion difference between both components in a CPM pair. For simplicity we assumed (1) a circular orbit in the plane of the sky, (2) an orbital radius corresponding to the projected physical separation (in AU) that can be computed as the product of the angular separation between both components (in arcsec) and the assumed common distance (in pc), and (3) a total mass of the system of 1.5 solar masses. With these assumptions, and following Kepler's third law, we can compute the orbital period and velocity and transform the latter to a proper motion, again using the known distance. This proper motion effect due to orbital motion, pmo, is the expected maximum proper motion difference between the components of a nearby wide binary. This effect increases with smaller distances and separations. For a wide binary located at $25 \mathrm{pc}$ from the Sun, separations of $3600 \mathrm{arcsec}, 60 \mathrm{arcsec}$, and 6 arcsec lead to pmo of about $1 \mathrm{mas}_{\mathrm{yr}}^{-1}, 8 \mathrm{mas} \mathrm{yr}^{-1}$, and $25 \mathrm{mas} \mathrm{yr}^{-1}$, respectively. With equal separations, the corresponding pmo are already about 4 times larger at $10 \mathrm{pc}$ and about 11 times larger at $5 \mathrm{pc}$, respectively.

Before we used pmo as a criterion for our CPM search in TGAS+UCAC5 data (see Sect. 3), we investigated the known CPM pairs in the TGAS $25 \mathrm{pc}$ sample alone. Here we have the advantage that we can find physical pairs of stars from their common distance and small angular separation and then check the agreement in their proper motions. As the TGAS proper motion errors are typically very small $\left(\ll 2\right.$ mas $\left.\mathrm{yr}^{-1}\right)$ and can be neglected, we used in this case the total proper 


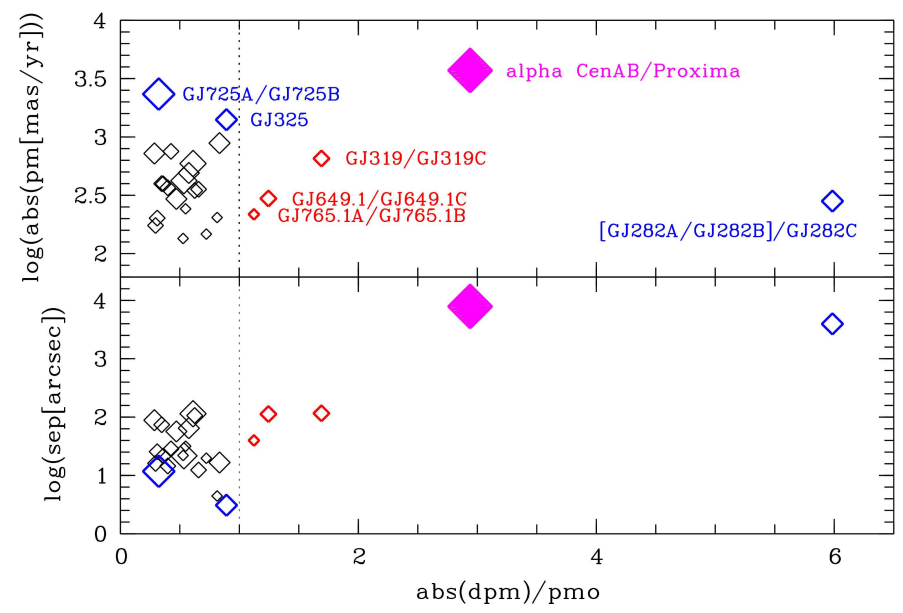

Fig. 1. Wide binaries and multiple systems with common parallaxes and relatively small angular separations (see text) in the TGAS $25 \mathrm{pc}$ sample. Shown are their total proper motions (top) and angular separations (bottom) as a function of the ratio of the absolute value of their total proper motion difference d pm (see Eq. (2)) to the estimated effect caused by orbital motion ( $p m o$ ). The dotted line indicates the expected maximal ratio of 1 . Systems discussed in the text are labelled in the top panel and indicated by thick red and blue open symbols. For comparison we added the values for Proxima with respect to alpha CenAB (filled magenta symbols), which are not included in TGAS. The symbol sizes increase with parallax.

motion differences (Eq. (2)), which also leads to a clearer presentation in Fig. 1. However, in our TGAS+UCAC5 CPM search (Sect. 3), we applied the pmo criterion to the individual proper motion components (in RA and Dec) taking into account their typically larger (and sometimes rather different) UCAC5 errors.

We searched for wide binaries and multiple systems within the TGAS $25 \mathrm{pc}$ sample with angular separations of up to several degrees. First, we allowed only for parallaxes that agree within their TGAS errors and found 18 CPM pairs with total proper motion differences smaller than pmo. Their angular separations ranged between 4-120 arcsec, their parallaxes between 45-114 mas, and their total proper motions between 130-900 mas $\mathrm{yr}^{-1}$. Allowing for two times larger proper motion differences, we found three more CPM pairs falling in the same ranges of angular separations, parallaxes, and total proper motions, respectively. These three CPM pairs correspond to well-known systems of G-type stars (GJ 765.1A/GJ 765.1B), $\mathrm{K}$ dwarfs (GJ649.1(=CCDM J16548+4722AB)/GJ649.1C), and early-M dwarfs (GJ 319(=CCDM J08427+0933AB)/GJ 319C), respectively (thick red open symbols in the central parts of the two panels of Fig. 1). Finally, as shown in Fig. 1, we arrived at 24 CPM systems, when we conservatively allowed for $10 \%$ differences in the TGAS parallaxes. Interestingly, all the latter three additional cases fall outside of at least one of the above given ranges and occupy edge regions in the two panels of Fig 1 (objects indicated by thick blue open symbols). One of these pairs (GJ $325=$ CCDM J08555+7048AB) consists of two early-M dwarfs with a very small angular separation of 3 arcsec

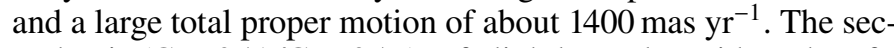
ond pair (GJ 725A/GJ 725B), of slightly cooler mid-M dwarfs, has also a relatively small angular separation of 11 arcsec, a large parallax of $>280$ mas, and very large total proper motion of about

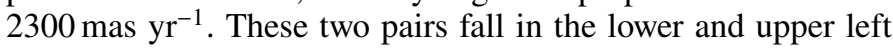
parts of the two panels in Fig. 1, respectively.
The third system, consisting of three TGAS sources, the wide binary GJ 282A/GJ 282B, and its much wider companion GJ $282 \mathrm{C}$ (shown by one symbol at the right edge of the top and bottom panels of Fig. 1, respectively), does not stand out by its parallax or proper motion but has an extremely large angular separation of about 3900 arcsec. This is much larger than the angular separations of all other wide binaries in the TGAS $25 \mathrm{pc}$ sample. At a distance of about $14 \mathrm{pc}$ it leads to a projected physical separation of about $56000 \mathrm{AU}$, which is even larger than the about 10000 AU between alpha CenAB and Proxima. The early$\mathrm{M}$ dwarf GJ 282C was studied with respect to the K-type binary GJ 282A/GJ 282B and found to rank among the widest physical companions by Poveda et al. (2009). As our nearest neighbours, Proxima and alpha CenAB represent another prominent case of an extremely wide but bound system (Kervella et al. 2017); we added the corresponding values in Fig. 1. These objects are not in TGAS, so we used their data as listed in Kervella et al. (2017). For [GJ 282A/GJ 282B]/GJ 282C we find ratios between the total proper motion differences and our estimated proper motion effect pmo of about 6 . These ratios exceed even those of alpha CenAB/ Proxima. Such very large ratios may indicate that our simple assumptions for computing pmo are not correct and/or that these systems are in the process of dynamical disintegration (Poveda et al. 2009).

\subsection{The role of chance alignments}

The larger the separation between the components of a wide binary candidate and the smaller their assumed CPM, the more likely is a chance alignment of unrelated objects. The proper motion errors also play an important role in the correct identification of CPM pairs, in particular if they are of the same order as the expected proper motion differences due to orbital motion (Sect. 2.2) and/or if they are not much smaller than the proper motion values. Lépine \& Bongiorno (2007) investigated CPM companions of HIPPARCOS primaries in the LSPM-North catalogue and excluded chance alignments using the following empirical relation between the separation ( $s e p)$, total proper motion difference $(\mathrm{d} p m)$, and total proper motion $(\mathrm{pm})$ :

$$
\frac{s e p * \mathrm{~d} p m}{\left(p m / p m_{\min }\right)^{3.8}}<1
$$

where sep was given in arcsec, whereas $\mathrm{d} p m$ and $\mathrm{pm}$ were in arcsec $\mathrm{yr}^{-1}$ here (but are in mas $\mathrm{yr}^{-1}$ throughout this paper), and $p m_{\min }$ was set to $0.15 \operatorname{arcsec} \mathrm{yr}^{-1}$, the lower proper motion limit of the LSPM-North catalogue. The total proper motion difference was computed as

$\mathrm{d} p m=\sqrt{\left(\mathrm{d} p m \mathrm{RA}^{2}+\mathrm{d} p m \mathrm{Dec}^{2}\right)}$.

In our search for new WD companions (Sect. 3), we applied several criteria, starting with a lower proper motion limit, selecting a maximum angular separation (similar to the limit used by Lépine \& Bongiorno 2007), taking into account the proper motion errors and the ratio of the proper motion differences to the pmo of each CPM candidate. In our final selection we considered only ratios below 1.5 such that we may have excluded some possible extremely wide binaries, similar to the cases described in Sect. 2.2. For an evaluation of our new WD CPM candidates and known WD CPM companions we checked, whether they fulfil the condition of Eq. (1). 


\section{Nearby TGAS/UCAC5 WD companion candidates}

\section{1. $C P M$ search criteria}

Our cross-matching of the TGAS 25 pc sample with the UCAC5 with a large search radius of 3600 arcsec yielded more than 7 million UCAC5 stars, on average about 7200 field stars per TGAS star. For each field star, we determined the pmo effect that we expected if it were a wide binary companion of the TGAS star (see Sect. 2.2). When we consider the proper motion differences dpm (TGAS-UCAC5) of potential CPM pairs, we must also take into account the proper motion errors epm in the TGAS $25 \mathrm{pc}$ sample and in UCAC5. Even after excluding UCAC5 stars with large proper motion errors, the TGAS proper motions were still about 10 times more accurate than those of the remaining UCAC5 stars. This is because the TGAS 25 pc sample consists mostly of HIPPARCOS stars, for which the TGAS was much more accurate than for Gaia stars. Only the 120 non-HIPPARCOS stars in the TGAS $25 \mathrm{pc}$ sample show a similar peak in the proper motion error distribution as in UCAC5. However, the tail of their error distribution contains only about 10 stars with errors

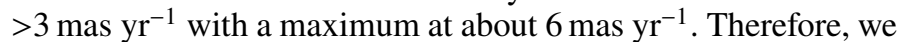
did not exclude potential CPM primaries based on TGAS proper motion errors. We selected candidate TGAS/UCAC5 CPM pairs with the following criteria:

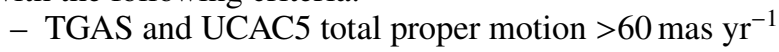

- TGAS-UCAC5 separation $<1800 \operatorname{arcsec}$

- epm ${ }_{U C A C 5}<6$ mas yr $^{-1}$ (in RA and Dec components)

- $|\mathrm{d} p m|<1.5 *\left(p m o+e p m_{T G A S}+e p m_{U C A C 5}\right)$ (in RA and Dec)

When we tried to change the first three limits towards smaller total proper motions, larger separations, and larger UCAC5 proper motion errors, respectively, the number of CPM candidates increased. However, as their total proper motions were generally smaller, it happened that several CPM candidates were found for a given TGAS primary, in particular for very nearby TGAS stars. The additional candidates also showed unrealistic absolute magnitudes (assuming a common distance with the primary) with their given colours in near-infrared and optical to near-infrared colour-magnitude diagrams (CMDs). The factor of 1.5 in the fourth selection criterion was a compromise justified by the fact that several known wide binaries among the nearby CPM pairs in TGAS alone and in TGAS/UCAC5 would have been excluded with a factor of 1.0, whereas factors of 2.0-2.5 led again to more doubtful candidates.

After applying the first selection criterion, the number of CPM candidates reduced to about $86000(\approx 1 \%$ of the original number of selected field stars). With the following two criteria, it further reduced to about 22000 and 11000 , respectively. The fourth and decisive criterion led to only $72(\approx 0.001 \%)$ CPM candidates, most of which turned out to be main sequence (MS) stars (see Figs. 2 and 3).

\subsection{WD companion candidates in CMDs}

After selecting only those 871 with accurate 2MASS photometry among the 973 stars of the TGAS 25 pc sample, the near-infrared CMD in Fig. 2 (black plus signs) shows the MS populated from (a few late-F and) early-G to early-M spectral types and a small WD sequence of four isolated WDs, WD 1142-645 (DQ6.4), WD 1647+591 (DAV4.1), WD 1917-077 (DBQZ4.9), and WD 2117+539 (DA3.6), as already mentioned by Tremblay et al. (2017) (spectral types are taken from Holberg et al. 2016). Figure 2 also shows the CPM companions identified in our search (filled red diamonds), where we assume that the parallax of a CPM companion is the same

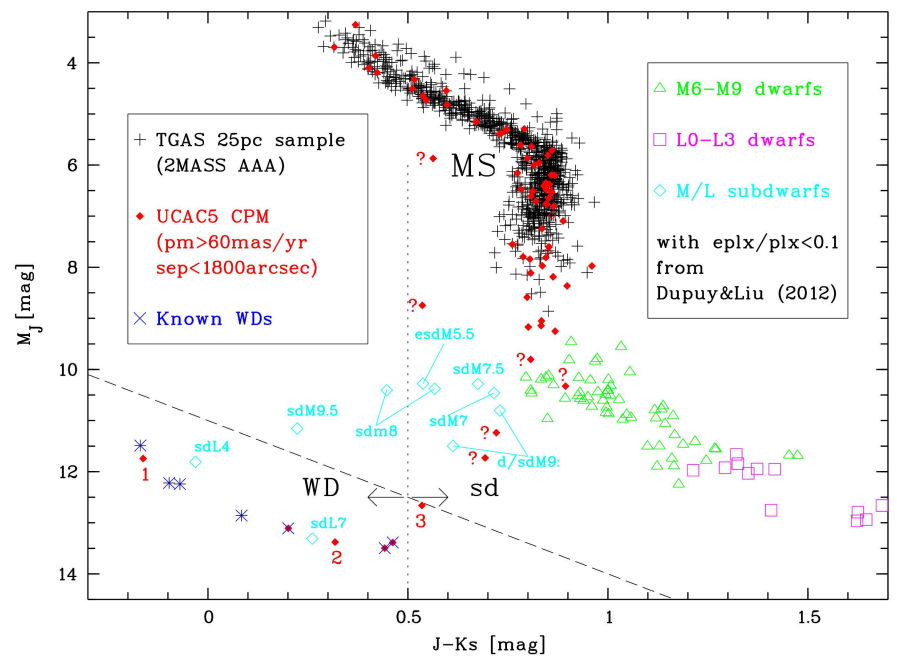

Fig. 2. Near-infrared 2MASS CMD for stars in the TGAS $25 \mathrm{pc}$ sample with photometric quality 'AAA' in the 2MASS catalogue (black plus signs) and for selected CPM companions (see text) from UCAC5 (filled red diamonds). Their absolute magnitudes $M_{J}$ are based on TGAS parallaxes. Four known WDs in TGAS and three known WD companions in UCAC 5 are marked by blue crosses. Our WD candidates (see Eqs. (3) and (4)) fall below both dashed lines in this figure and in Fig. 3, respectively. The dotted line shows the dividing line at $J-K_{s}=+0.5$ between WDs and cool subdwarfs (sd) found by Subasavage et al. (2017). Also shown are normal (not flagged as atypical, and not known as close binaries) late-M (green open triangles), and early-L dwarfs (magenta open squares) as well as late-M and L subdwarfs (cyan open diamonds), indicated by their spectral types, from Dupuy \& Liu (2012) based on their parallaxes with relative errors less than $10 \%$. Three new WD companion candidates are numbered. All CPM candidates with angular separations $>900$ arcsec are labelled with question marks.

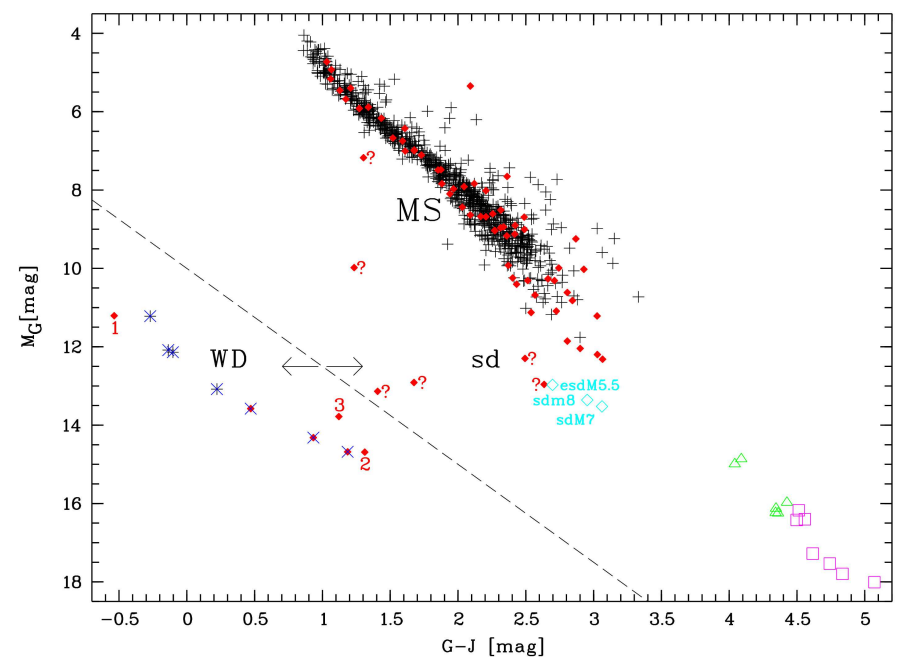

Fig. 3. Optical (Gaia) to near-infrared (2MASS) CMD with the same stars (and symbols) as in Fig. 2. Only part of the late-M, early-L dwarfs, and M/L subdwarfs from Dupuy \& Liu (2012) could be identified in Gaia (see text). As in Fig. 2, the absolute Gaia magnitudes $M_{G}$ of TGAS stars and UCAC5 CPM candidates are based on TGAS parallaxes, whereas those of the comparison objects are based on the parallaxes from Dupuy \& Liu (2012). Objects located below the dashed lines in this figure and in Fig. 2 were considered as WDs.

as that of its TGAS primary. With our CPM companion search aiming at WD companions, we did not expect to find late-M dwarfs (and brown dwarfs with late-M, L, or T spectral types) with $M_{J}>10$, as even the nearest of these objects are too faint 
to be included in UCAC5. Consequently, the UCAC5 CPM companions only slightly extend the MS towards fainter magnitudes. For comparison we show in Fig. 2 that late-M and early-L dwarfs occupy the lower right part of the near-infrared CMD as derived from accurate parallaxes by Dupuy \& Liu (2012).

From nine known WD companions of TGAS stars within $25 \mathrm{pc}$ (Tremblay et al. 2017), only three of these companions are included in UCAC5 (WD 0433+270, WD 0751-252, and WD 2154-512). All three of these companions were found by our CPM selection criteria. They extend the small WD sequence in Fig. 2 (red filled diamonds overplotted by blue crosses) to the red. Their angular separations range between 28 arcsec and 400 arcsec. The other six WD companions that are not in UCAC5 have smaller angular separations (7-17 arcsec) from their bright primaries, which may have prevented their detection on the UCAC images; two of these WD companions may also have been too faint $(V>16)$ to be observed within the UCAC survey.

The data of known and candidate nearby $(d<25 \mathrm{pc})$ TGAS/UCAC5 CPM pairs including a WD companion, all found with the selection criteria described in Sect. 3.1, are listed in the upper section of Table 1. Two of the three new WD companion candidates have relatively small angular separations, whereas the third, HD $35650 \mathrm{~B}$, looks with its large angular separation at approximately the same distance similar to the known very wide CPM companion SCR J0753-2524. Consequently, in these two pairs the pmo effect is relatively small compared to the proper motion errors.

From Fig. 2 it is obvious that the WD companion candidates " 1 " and " 2 " fit well into the sequence of known WDs, whereas candidate " 3 " lies a bit off and just right of the WD/subdwarf dividing line described by Subasavage et al. (2017). Doubtful candidates indicated with "?", which had the largest angular separations $(\approx 1000-1750$ arcsec $)$ but relatively small (60-90 mas $\left.\mathrm{yr}^{-1}\right)$ total proper motions, are in this nearinfrared CMD either much redder or brighter than the WD sequence. However, because of their small proper motions and spectral classification (in SIMBAD) of their apparent primaries as normal MS stars, we cannot consider these doubtful CPM companions as nearby subdwarf candidates. With their large separations, it is much more likely that these are chance alignments of distant MS stars (with distances in the range of $\approx 50-500 \mathrm{pc}$ according to their $G-J$ colour and apparent $G$ magnitudes) with the nearby TGAS stars. In fact, if we compute the ratio according to Eq. (1), replacing the $p m_{\min }$ used by Lépine \& Bongiorno (2007) with our lower proper motion limit $p m_{\text {min }}=0.06$ arcsec $\mathrm{yr}^{-1}$, we obtain for these six doubtful CPM pairs values between 1.4 and 5.3, indicating chance alignments. The tangential velocities derived from the proper motions and the estimated larger photometric distances of these rejected CPM companions are mostly in the range of $20-80 \mathrm{~km} \mathrm{~s}^{-1}$ that is typical of the Galactic thin disk population. The resulting tangential velocity was larger $\left(\approx 150 \mathrm{~km} \mathrm{~s}^{-1}\right)$ for one object alone, which can be identified as 2MASS J06233146-5952448 (located just above the dashed line in Fig. 3), such that this object might be a distant (K-type) subdwarf (thick disk object) unrelated to our solar neighbourhood TGAS sample.

For candidate " 3 ", this ratio is also large (2.1) indicating a probable chance alignment. However, its separation is only moderately large (about 465 arcsec) and its location in Fig. 3 lies much closer to the WD sequence. Therefore, we consider this object as a weak WD companion candidate. Interestingly, its relatively blue colour $(G-J \approx+1.1)$ and faint magnitude $(G \approx 15)$ lead to an alternative photometric distance of $\approx 800 \mathrm{pc}$ and tangential velocity of $\approx 260 \mathrm{~km} \mathrm{~s}^{-1}$, if it is unrelated. In this case, this would be a distant G-type subdwarf candidate, possibly belonging to the Galactic halo population. For all other CPM candidates, including known WD companions and the WD companion candidates 1 and 2, the ratio from Eq. (1) was always smaller than 0.4 , with a mean value of 0.02 , confirming their real CPM status.

Concerning the location of candidate 3 close to the WD/subdwarf dividing line (dotted line in Fig. 2), we note that the $J-K_{s}$ colours used in Subasavage et al. (2017) were not directly measured in but were transformed to the 2MASS system. The position of this dividing line is questionable in view of the widespread $J-K_{s}$ colour distribution of the shown M/L subdwarfs and obviously not valid for the latest type (coolest) known subdwarfs, SSSPM J1013-1356 (sdM9.5), 2MASS J16262034+3925190 (sdL4), and 2MASS J05325346+8246465 (sdL7). The remaining seven M/L subdwarfs seem to represent better the $60 \mathrm{pc}$ cool subdwarf sample (presumably dominated by $\mathrm{sdK}$ and early-sdM subdwarfs) that was used by Subasavage et al. (2017) in finding the $J-K_{s}=+0.5$ dividing line. We considered objects as WD candidates if they were falling below two colour-magnitude limits (shown by the dashed lines in Figs. 2 and 3)

$M_{G}>10.0+(2.5 *(G-J))$,

$M_{J}>11.0+\left(3.0 *\left(J-K_{S}\right)\right)$.

Contrary to the WD/subdwarf confusion in a near-infrared $M_{J} / J-K_{s}$ diagram (Fig. 2), we expect a clearer separation of the MS, subdwarf, and WD sequences in an optical to nearinfrared CMD. As Gaia provides accurate magnitudes, albeit in a very broad optical band, we studied the TGAS $25 \mathrm{pc}$ sample and our UCAC5 CPM candidates together with the comparison objects from Dupuy \& Liu (2012) in an $M_{G} / G-J$ diagram (Fig. 3). Unfortunately, only 3 out of $10 \mathrm{M} / \mathrm{L}$ subdwarfs could be identified in Gaia DR1 (with Gaia magnitudes $16.3<G<17.7$ ). This low identification rate may be due to the very large proper motions of these ultracool subdwarfs, which are probably problematic for Gaia DR1. Among the comparison late-M and early-L dwarfs, we identified 6 out of 58 (only $10 \% ; 15.8<G<17.7)$ and 7 out of $12(58 \% ; 18.5<G<$ 20.6) objects in Gaia DR1, respectively. The very low identification rate of the relatively bright and nearby late-M dwarfs may be caused by their large parallaxes and proper motions and selection criteria for problematic sources in Gaia DR1. The much fainter early-L dwarfs have typically smaller parallaxes and proper motions so that their identification rate is similar to that found for all $\mathrm{L}$ and $\mathrm{T}$ dwarfs in Gaia DR1 (45\%; Smart et al. 2017).

The MS in Fig. 3 shows a large gap at the expected position of early- to mid-M dwarfs. Three labelled late-M subdwarfs form a parallel sequence shifted from the MS by $\approx 0.5$ mag to the blue. Some of the doubtful CPM candidates marked with "?" seem to fall in the same region, but we consider these candidates as chance alignments rather than nearby subdwarf candidates (see above). The WD sequence is also almost parallel with respect to the MS, but shows a large blue-shift of $\approx 3.0 \mathrm{mag}$. The new WD candidates 1 and 2 are now located at the blue and red end of the WD sequence, suggesting a hot and a cool WD, respectively. The two L-type subdwarfs, which fell right in the WD sequence in the near-infrared CMD (Fig. 2) have no Gaia magnitudes yet, but we expect these subdwarfs to fall in the lower right corner of Fig. 3, well separated from the WDs in $G-J$ colour. 
Table 1. Astrometry and photometry of known and candidate (1-3) WD companions and their primaries in nearby TGAS/UCAC5 CPM pairs and of additional known WDs with no previous trigonometric parallaxes (in SIMBAD) and one WD candidate (4) found in the UPC.

\begin{tabular}{|c|c|c|c|c|c|c|c|c|c|c|c|c|}
\hline $\begin{array}{l}\text { Name } \\
\text { (WD name/cand) }\end{array}$ & [deg] & $\begin{array}{l}\operatorname{Dec}^{a} \\
{[\mathrm{deg}]}\end{array}$ & $\begin{array}{c}p l x_{T G A S} \\
(\mathrm{Sep}, \mathrm{PA})^{a} \\
{[\mathrm{mas}]} \\
\left(\left[\operatorname{arcsec},{ }^{\circ}\right]\right)\end{array}$ & $\begin{array}{l}p l x_{U P C} \\
{[\mathrm{mas}]}\end{array}$ & $\begin{array}{r}p m \mathrm{RA} \\
{\left[\text { mas } \mathrm{yr}^{-1}\right]}\end{array}$ & $\begin{array}{c}p m \text { Dec } \\
{\left[\text { mas } \mathrm{yr}^{-1}\right]}\end{array}$ & $\begin{array}{c}p m o \\
(|\mathrm{~d} p m|)^{f} \\
{\left[\mathrm{mas} \mathrm{yr}^{-1}\right]} \\
\left(\left[\mathrm{mas} \mathrm{yr}^{-1}\right]\right)\end{array}$ & {$[\mathrm{mag}]$} & $\begin{array}{c}J^{b} \\
{[\mathrm{mag}]}\end{array}$ & $\begin{array}{l}J-K_{s}{ }^{b} \\
{[\mathrm{mag}]}\end{array}$ & SpT & Ref \\
\hline GJ $171.2 \mathrm{~A}$ & 069.202096 & +27.131577 & $57.2 \pm 0.3$ & $65.1 \pm 9.0$ & $+232.1 \pm 0.1^{c}$ & $-147.7 \pm 0.1^{c}$ & 9 & 7.64 & 5.95 & +0.71 & K3IVke & 3 \\
\hline $\begin{array}{l}\text { GJ } 171.2 \mathrm{~B} \\
(=\mathrm{WD} 0433+270)\end{array}$ & 069.188131 & +27.163694 & $(124.0,339)$ & $80.1 \pm 11.0$ & $\begin{array}{l}+230.8 \pm 3.0^{d} \\
+229.7 \pm 6.1^{e}\end{array}$ & $\begin{array}{l}-146.6 \pm 2.9^{d} \\
-142.9 \pm 6.0^{e}\end{array}$ & $(1,1)$ & 15.53 & 14.60 & +0.46 & DA9.0 & 1 \\
\hline Ross 429 & 118.543958 & -25.302335 & $56.2 \pm 0.3$ & - & $-300.8 \pm 0.1^{c}$ & $+201.0 \pm 0.1^{c}$ & 5 & 8.97 & 7.02 & +0.85 & M0 & 4 \\
\hline $\begin{array}{l}\text { SCR J0753-2524 } \\
(=\text { WD 0751-252) }\end{array}$ & 118.484536 & -25.399551 & $(399.8,209)$ & - & $-286.4 \pm 5.2^{d}$ & $+205.5 \pm 5.3^{d}$ & $(14,4)$ & 15.93 & 14.75 & +0.44 & DA9.8 & 1 \\
\hline GJ $841 \mathrm{~A}$ & 329.421448 & -51.007760 & $66.1 \pm 0.5$ & - & $-35.3 \pm 0.2^{c}$ & $-379.8 \pm 0.2^{c}$ & 25 & 9.24 & 6.75 & +0.87 & $\mathrm{M} 2 \mathrm{Ve}$ & 5 \\
\hline $\begin{array}{l}\text { GJ } 841 \text { B } \\
(=W D 2154-512)\end{array}$ & 329.409514 & -51.010412 & $(28.7,251)$ & - & $-46.5 \pm 1.2^{d}$ & $-395.5 \pm 1.1^{d}$ & $(11,16)$ & 14.48 & 14.01 & +0.20 & DQ8.3 & 1 \\
\hline HD 166435 & 272.339436 & +29.951968 & $40.7 \pm 0.2$ & $46.1 \pm 8.8$ & $+71.9 \pm 0.1^{c}$ & $+61.1 \pm 0.1^{c}$ & 12 & 6.62 & 5.69 & +0.37 & G1IV & 3 \\
\hline $\begin{array}{l}\text { HD } 166435 \text { B } \\
\left(={ }^{\prime} 1 \text { ') }\right.\end{array}$ & 272.331393 & +29.956100 & $(29.2,301)$ & $50.4 \pm 3.3$ & $\begin{array}{l}+64.8 \pm 1.2^{d} \\
+67.4 \pm 2.4^{e}\end{array}$ & $\begin{array}{l}+72.4 \pm 1.2^{d} \\
+71.5 \pm 2.4^{e}\end{array}$ & $(7,11)$ & 13.16 & 13.70 & -0.16 & $\mathrm{DA} 2.2 \pm 0.2$ & 2 \\
\hline TYC 3980-1081-1 & 327.909017 & +59.294450 & $120.6 \pm 1.0$ & $154.7 \pm 12.1$ & $-70.8 \pm 2.5^{c}$ & $+76.1 \pm 2.4^{c}$ & 84 & 9.20 & 6.53 & +0.88 & $\approx \mathrm{M} 2$ & 2 \\
\hline $\begin{array}{l}\text { TYC } 3980-1081-1 \text { B } \\
\left(={ }^{\prime} 2 '\right)\end{array}$ & 327.916408 & +59.292935 & $(14.6,112)$ & $131.0 \pm 4.4$ & $\begin{array}{l}-87.2 \pm 2.2^{d} \\
-79.5 \pm 3.3^{e}\end{array}$ & $\begin{array}{l}-31.2 \pm 2.0^{d} \\
-17.3 \pm 3.2^{e}\end{array}$ & $(16,107)$ & 14.28 & $12.97^{g}$ & $+0.32^{g}$ & $\approx \mathrm{DA} 10 ?$ & 2 \\
\hline $\begin{array}{l}\text { HD } 35650 \\
\text { HD } 35650 \text { B } \\
(=` 3 ’) \\
\end{array}$ & $\begin{array}{l}081.125938 \\
081.252385\end{array}$ & $\begin{array}{l}-38.969890 \\
-39.053688\end{array}$ & $\begin{array}{c}57.4 \pm 0.3 \\
(464.9,131)\end{array}$ & - & $\begin{array}{l}+43.1 \pm 0.1^{c} \\
+46.3 \pm 1.9^{d}\end{array}$ & $\begin{array}{l}-57.3 \pm 0.1^{c} \\
-48.8 \pm 2.0^{d}\end{array}$ & $\begin{array}{c}5 \\
(3,9)\end{array}$ & $\begin{array}{r}8.43 \\
14.99\end{array}$ & $\begin{array}{c}6.70 \\
13.87\end{array}$ & $\begin{array}{l}+0.78 \\
+0.53\end{array}$ & $\begin{array}{l}\text { K6Vke } \\
\approx \text { DA9? }\end{array}$ & $\begin{array}{l}6 \\
2\end{array}$ \\
\hline $\begin{array}{l}\text { GJ } 3285 \\
(=W D 0423+120\end{array}$ & 066.473476 & +12.195784 & - & $58.0 \pm 8.0$ & $-94.6 \pm 6.4^{e}$ & $-227.9 \pm 6.4^{e}$ & - & 15.21 & 14.49 & +0.24 & DC8.2 & 1 \\
\hline $\begin{array}{l}\text { GJ } 275.2 \mathrm{~B}^{h} \\
(=\mathrm{WD} 0727+482\end{array}$ & 112.695736 & +48.168756 & - & $89.6 \pm 4.2$ & $-216.6 \pm 4.0^{e}$ & $-1274.9 \pm 3.8^{e}$ & - & 14.84 & 13.08 & +0.33 & DA10.0+DA10.1 & 1 \\
\hline $\begin{array}{l}\text { GJ } 1098 \\
(=\text { WD } 0728+642\end{array}$ & 113.379072 & +64.156548 & - & $54.3 \pm 8.2$ & $+50.9 \pm 6.8^{e}$ & $-260.5 \pm 5.9^{e}$ & - & 15.91 & 14.81 & +0.43 & DA11.1 & 1 \\
\hline $\begin{array}{l}\text { GJ } 4165^{j} \\
(=\text { WD } 2047+372\end{array}$ & $312.278706^{e}$ & $+37.471154^{e}$ & - & $63.1 \pm 2.9$ & $+167.1 \pm 2.9^{e}$ & $+154.3 \pm 2.9^{e}$ & - & - & 13.30 & -0.13 & DA3.6 & 1 \\
\hline $\begin{array}{l}\text { UPC } 72924 \\
\left(={ }^{\prime} 4^{\prime}\right)\end{array}$ & 277.995093 & +46.974870 & - & $40.2 \pm 6.6$ & $+61.5 \pm 4.6^{e}$ & $+38.4 \pm 4.4^{e}$ & - & 15.05 & 14.52 & +0.14 & $\approx$ DA7? & 2 \\
\hline
\end{tabular}

Notes. Gaia coordinates are for (J2000, epoch 2015.0) and were rounded to 0.000001 degrees. Although the TGAS data may be more accurate, all parallaxes and their errors were rounded to 0.1 mas, proper motions and their errors to 0.1 mas $\mathrm{yr}^{-1}$. The absolute values of measured proper

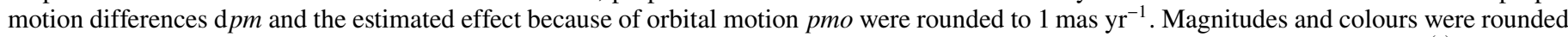
to $0.01 \mathrm{mag}$. Spectral types with question marks are only guesses based on $G-J$ and $M_{G}$ (Figs. 3 and 5). Further notes on the data: ${ }^{(a)} G a i a$ DR1, ${ }^{(b)}$ 2MASS, ${ }^{(c)}$ TGAS, ${ }^{(d)}$ UCAC5, ${ }^{(e)} \mathrm{UPC},{ }^{(f)}$ two values are given for RA and Dec, respectively, ${ }^{\left({ }^{g}\right)} J$ magnitude with very poor goodness-of-fit quality of the profile-fit photometry, ${ }^{(h)} \mathrm{An}$ accurate parallax of $87.4 \pm 0.5$ mas measured for this close binary by Nelan et al. (2015), ${ }^{(i)}$ Subasavage et al. (2009) measured a parallax of $56.54 \pm 0.95$ mas, ${ }^{(j)}$ this object is not plotted in Figs. 4 and 5, since it was not identified in Gaia DR1.

References. (1) Holberg et al. (2016); (2) this paper; (3) Gray et al. (2003); (4) Gaidos et al. (2014); (5) Torres et al. (2006); (6) Gray et al. (2006).

We note that all the $\mathrm{M} / \mathrm{L}$ subdwarfs from Dupuy \& Liu (2012) are fainter than the UCAC5 magnitude limit of $R \approx 16$ mag. The same is true for the MS late-M and early- $\mathrm{L}$ dwarfs. As already discussed above, the Gaia magnitude intervals for the M/L subdwarfs and the MS late-M/early-L dwarfs are almost identical and their UCAC5 magnitudes fall in the same range. With our quality cut for UCAC5 proper motion errors, we effectively reduced the limiting UCAC5 magnitude of the investigated CPM candidates further. The $\mathrm{M} / \mathrm{L}$ subdwarfs have in addition very large proper motions (between $\approx 600 \mathrm{mas} \mathrm{yr}^{-1}$

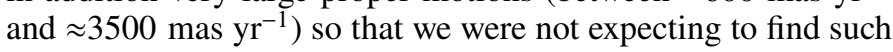
objects in our CPM search aiming at relatively small proper motions. The region between WDs and cool subdwarfs in Fig. 3 should be empty. Therefore, all CPM candidates falling in this CMD close to the arbitrary drawn dividing line between WDs and subdwarfs have to be taken with caution, independent of their possible chance alignment that we evaluated using Eq. (1).

\section{UPC parallaxes confirming two WD companions}

The primaries of our WD companion candidates 1 and 2, HD 166435 and TYC 3980-1081-1, were only recently added to the
$25 \mathrm{pc}$ sample. The original HIPPARCOS (ESA 1997) parallax of the well-known early-G star HD 166435 placed it at about $25.2 \mathrm{pc}$, whereas the revised HIPPARCOS (van Leeuwen 2007) results led to a distance of $24.8 \mathrm{pc}$ and the TGAS parallax finally fixed it at 24.58 pc. Finch et al. (2014) discovered the Gaia star TYC 3980-1081-1 as a close neighbour of the Sun, estimating a photometric distance of $5.9 \mathrm{pc}$; this discovery occurred before a first trigonometric parallax from URAT data was reported by Finch \& Zacharias (2016b) giving a distance of about $6.5 \mathrm{pc}$. The full UPC catalogue, including all significant parallaxes determined from the complete northern hemisphere URAT data, was only recently made available (Finch \& Zacharias 2016a,c). The TGAS parallax of TYC 3980-1081-1 corresponds to a distance of $8.29 \mathrm{pc}$, still well within the $10 \mathrm{pc}$ limit. From its absolute magnitude of $M_{J}=6.94$ we estimate a spectral type of $\approx \mathrm{M} 2$ for TYC 3980-1081-1 using the relationship between absolute magnitudes and spectral types from Scholz et al. (2005).

Interestingly, not only HD 166435 and TYC 3980-1081-1 can be found in the UPC, but also their WD companion candidates 1 and 2, which we selected based on their CPM in TGAS and UCAC5. We call these HD 166435 B and TYC 3980-1081-1 $\mathrm{B}$, respectively. Their UPC parallaxes, albeit much less accurate 


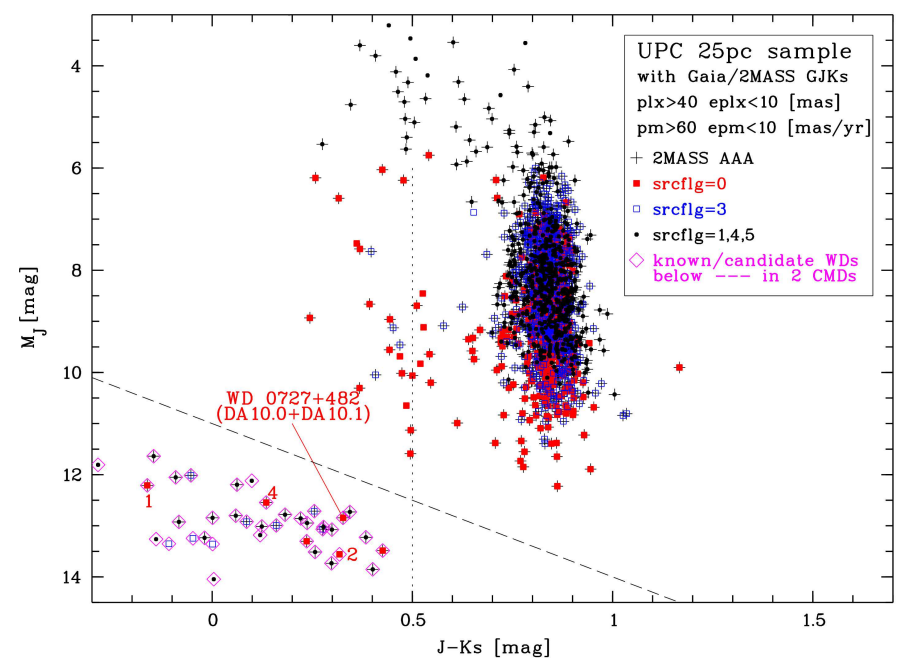

Fig. 4. Near-infrared (2MASS) CMD for our selected UPC $25 \mathrm{pc}$ sample. Absolute magnitudes are based on UPC parallaxes. The start and end values of both axes are the same as in Fig. 2, the dashed line shows our previously chosen limit for the WD region in this CMD (Eq. (3)), the dotted line again indicates the WD/subdwarf dividing line of Subasavage et al. (2017). Stars with photometric quality 'AAA' in 2MASS are plotted as plus signs. Overplotted as red filled squares, blue open squares, and black dots are stars without previous parallax measurements (UPC srcflg $=0$ ), with already published data in Finch \& Zacharias (2016b) $(\operatorname{srcflg}=3)$, and in the literature ( $\operatorname{srcflg}=1,4,5)$, respectively. All WD candidates, defined by us as falling below the dashed lines in this figure and in Fig. 5, are shown as open diamonds. The new WD candidates 1 and 2 (already found in our TGAS CPM search in UCAC5), and 4 are indicated. A close binary cool WD is also labelled.

than the TGAS parallaxes, are similarly large (see upper section of Table 1) as for the primaries and confirm a common distance, respectively. This is important in particular for our second candidate, TYC 3980-1081-1 B, which shows a large difference in the Dec proper motion component compared to that of TYC 3980-1081-1. However, the close distance and small separation of this CPM pair lead to a very large pmo value and the proper motion difference is not much larger than this expected effect from orbital motion. In case of the known wide binary Ross 429/ SCR J0753-2524, discovered by Subasavage et al. (2005), the proper motion differences are also relatively large with respect to the corresponding pmo value (Table 1). In our fourth selection criterion described in Sect. 3.1 we took also into account the proper motion errors, which are relatively large for the UCAC5 proper motion of SCR J0753-2524.

The UPC proper motions of three CPM companions, also given in the upper section of Table 1, are less accurate than their UCAC5 proper motions. However, for the two relatively bright WD companions, HD 166435 B and TYC 3980-1081-1 B, the combined parallax and proper motion solution in the UPC led to more similar proper motions compared toAstrometry and photometry of known their primaries, respectively. For the fainter known companion WD $0433+270$ the UPC errors of its parallax and proper motion are much larger and the UCAC5 proper motion is in better agreement with the TGAS proper motion of the primary than the UPC proper motion.

\section{Additional WD candidates from the UPC}

Finch \& Zacharias (2016b) have already studied stars falling in a $25 \mathrm{pc}$ sample according to significant parallax measurements

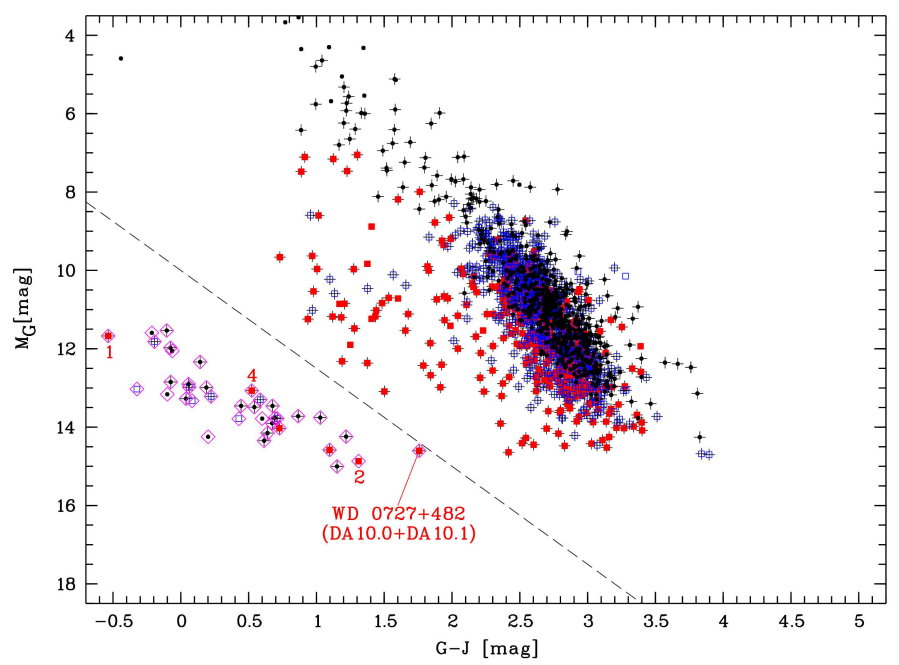

Fig. 5. Optical (Gaia) to near-infrared (2MASS) CMD for our selected UPC 25 pc sample. As in Fig. 4, absolute magnitudes are based on UPC parallaxes. The start and end values of both axes are the same as in Fig. 3; the dashed line again shows our limit for the WD region in this CMD (Eq. (4)). Symbols are the same as in Fig. 4.

in the UPC. These authors mentioned many new nearby stars with small proper motions $\left(<200\right.$ mas $\left.\mathrm{yr}^{-1}\right)$ but did not pay special attention to potential WDs among their new neighbour candidates. In their notes on individual systems these authors discussed the nearest new discoveries and found many of these discoveries to be suspicious with respect to blended or elongated images and/or large scatter in the combined fit for parallax and proper motion. These suspicious nearest candidates have parallax and proper motion errors of typically $10-25$ mas and $10-20$ mas $\mathrm{yr}^{-1}$, respectively. From $\approx 112000$ stars in the full UPC available at the CDS (Finch \& Zacharias 2016 c), $\approx 5200$ have parallaxes $>40$ mas and for $\approx 3700$ of these the parallax errors are smaller than 10 mas. As the UPC covers only about half of the sky, this number appears very high in comparison with the already mentioned RECONS all-sky $25 \mathrm{pc}$ census, and the new UPC neighbours need to be taken with caution.

To evaluate potential WDs in near-infrared and optical to near-infrared CMDs, similar to those shown in Figs 2 and 3, we cross-matched the UPC with 2MASS and Gaia DR1. We found $\approx 3700$ UPC stars within $25 \mathrm{pc}$ that have both 2 MASS and Gaia counterparts. As expected from the two different parts of the UPC (stars with or without previously known parallaxes; see Finch \& Zacharias 2016a,c) the error distribution for the UPC parallaxes rises to a peak at 10 mas before it abruptly turns down to a long tail reaching to about 60 mas. The proper motion errors show a more symmetric distribution with a maximum at about

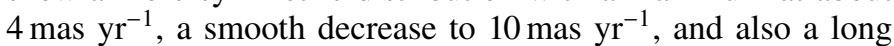

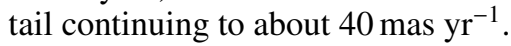

For our final UPC $25 \mathrm{pc}$ sample, consisting of $\approx 1600$ stars, we applied the following quality criteria concerning the parallax and proper motion errors, eplx and epm, respectively, and also used a lower limit for the total proper motion:

- eplx $x_{U P C}<10$ mas;

- epm ${ }_{U P C}<10$ mas $\mathrm{yr}^{-1}$ (in RA and Dec components);

- UPC total proper motion $>60$ mas $_{\mathrm{yr}^{-1}}$;

The resulting CMDs are shown in Figs. 4 and 5. As in Figs. 2 and 3, we plot stars with reliable 2MASS photometry as plus signs, but overplot all stars independent of their 2MASS quality with various symbols corresponding to their 
source flags $(\operatorname{srcflg})$ given in the UPC. Stars with $s r c f l g=0$, without previously published parallaxes in SIMBAD or other catalogues, are plotted as filled red squares (except for GJ 4165 lacking a $G$ magnitude) and are listed in the lower section of Table 1 if not already listed in the upper section. Because of the deeper limiting magnitude of URAT compared to UCAC5, the MS is now mainly populated with $\mathrm{M}$ dwarfs that were lacking in TGAS and TGAS/UCAC5 CPM data. With $M_{J}=6-11$, these are M0-M7 dwarfs according to Scholz et al. (2005). As expected from the lower accuracy of the UPC parallaxes in comparison to TGAS, the MS and the WD sequence in Figs. 4 and 5 do not look as narrow as in Figs. 2 and 3. In particular, many of the new UPC neighbours ( $\operatorname{srcflg}=0$ and $s r c f l g=3$ ) are located left of the MS, where only few subdwarfs are expected. However, all our WD candidates defined by Eqs. (3) and (4) are well separated from the cloud of suspicious subdwarfs left of the MS. The only WD located close to the corresponding dashed line in Fig. 3, WD $0727+482$, is a close binary, expected to be brighter. We also note that none of the 34 known WDs and 3 WD candidates 1, 2 and 4 in Fig. 4 falls right of the WD/subdwarf dividing line of Subasavage et al. (2017). The new candidate "4" is a previously anonymous field star, UPC 72924.

When we tried to allow for larger parallax and proper motion errors in the UPC $25 \mathrm{pc}$ sample, for example, 12 mas and

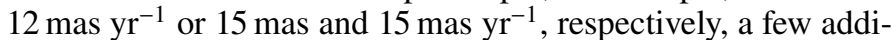
tional known WDs were recovered. These WDs all fell in the WD regions below the dashed lines in both CMDs, whereas new WD candidates were not found. On the other hand, a lower proper

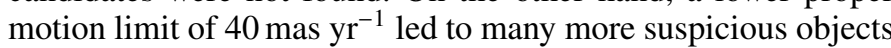
falling closer to the dashed lines in both CMDs but only one more WD candidate that we nevertheless considered as unreliable because of its relatively large image elongation and large scatter of the post-fit residuals given in the UPC.

We note that unless we did not search for CPM candidates in the UPC alone or with respect to TGAS, one of the known WDs with a UPC parallax in Table 1, WD $0727+482$, which is a close binary itself, is a member of a wide multiple system. This system includes GJ 275.2 A, which has a similarly large UPC parallax and proper motion of $101.2 \pm 4.8$ mas and $(-192.5 \pm 4.4$, $-1268.4 \pm 4.1)$ mas $\mathrm{yr}^{-1}$, respectively.

\section{One spectroscopically confirmed WD companion}

Our WD candidate HD 166435 B was already included in our earlier large programme concentrating on the spectroscopic classification of nearby cool WD and subdwarf candidates. Within this programme it was one of the targets with the smallest proper motion at that time selected from an earlier UCAC version. Large numbers of comparison objects with known spectral types were also observed. The low-resolution spectroscopic observations were carried out in 2008 (August 8/9) using the focal reducer and faint object spectrograph CAFOS mounted at the $2.2 \mathrm{~m}$ telescope at Calar Alto, Spain. We used the grism B 200 giving a wavelength coverage from about $3500 \AA$ to $7000 \AA$ and a dispersion of $4.7 \AA$ per pixel. All spectra were reduced with standard routines from the ESO MIDAS data reduction package.

Figure 6 shows the normalised spectrum of HD 166435 B together with those of two comparison objects observed during the same observing run. In this comparison HD 166435 B appears very similar to the DA2.4 and DA2.8 white dwarfs and could be classified as DA2.0 from its bluer continuum. The very blue continuum of HD 166435 B is consistent with its magnitudes and the resulting negative $F U V-N U V$

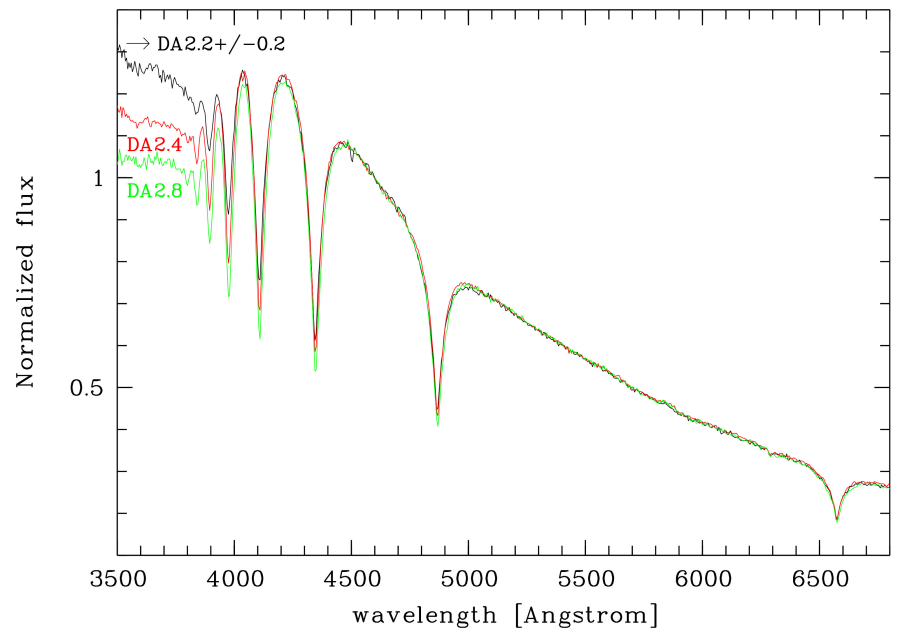

Fig. 6. Low-resolution Calar Alto $2.2 \mathrm{~m} / \mathrm{CAFOS}$ spectrum of candidate "1" (=HD 166435 B), indicated in Figs. 2 and 3, overplotted by the spectra of two known WDs, WD 2032+248 (DA2.4; red line), and WD 2149+021 (DA2.8; green line) (Gianninas et al. 2011), observed with the same instrument set-up. All spectra are normalised at $4600 \AA$.

colour index measured by the Galaxy Evolution Explorer (GALEX; Bianchi et al. 2011), $F U V=12.068 \pm 0.003$ and $N U V=12.735 \pm 0.004$, compared to $F U V=12.604 \pm 0.007$ and $N U V=12.688 \pm 0.005$ and a zero colour index for WD 2149+021 (WD 2032+248 was not measured by GALEX). The distances of HD $166435 \mathrm{~B}$ and WD 2149+021 are very similar with $\approx 24.6 \mathrm{pc}$ (TGAS) and $\approx 24.5 \mathrm{pc}$ (Limoges et al. 2015), respectively.

We also measured the equivalent widths of the $\mathrm{H} \beta$ and $\mathrm{H} \alpha$ lines, relative to their well-defined continuum in the three spectra of Fig. 6. Whereas our results for $\mathrm{H} \alpha$ support a slightly earlier classification of HD 166435 B (DA1.9) compared to WD 2032+248 (DA2.1) and WD 2149+021 (DA2.5), the equivalent widths of $\mathrm{H} \beta$, measured with higher signal to noise, hint at DA2.4, DA2.2, and DA2.7, respectively. We assign a preliminary spectral type of DA2.2 \pm 0.2 to HD $166435 \mathrm{~B}$.

For the other two WD companion candidates, TYC 39801081-1 B and HD 35650 B, we only assume cool DA types from their location in the optical to near-infrared CMD (Fig. 3) in comparison to the known WDs GJ 171.2 B and SCR J0753-2524 (Table 1). For the WD candidate UPC 72924 we assume a spectral type of $\approx$ DA7 from comparing its $G-J$ colour with those of known WDs found in our study of the UPC 25 pc sample.

\section{Conclusions}

We draw the following conclusions from our study of nearby $(d<25 \mathrm{pc})$ WDs in TGAS+UCAC5 and URAT data:

1. Compared to only four known WDs in TGAS and three known WD companions in UCAC5, our three new WD companion candidates represent an increase of $\approx 43 \%$.

2. Two of our new WD companion candidates are confirmed by parallax measurements in the UPC. One, HD 166435 B, orbits a G-type star at a distance of $\approx 24.6 \mathrm{pc}$ with a projected physical separation of $\approx 700 \mathrm{AU}$; the other, TYC 3980-1081-1 B, orbits an early-M dwarf at only $\approx 8.3 \mathrm{pc}$ distance with a projected physical separation of $\approx 120 \mathrm{AU}$. The third WD companion candidate, HD $35650 \mathrm{~B}$, has a very large projected physical separation from its primary of $\approx 8100 \mathrm{AU}$, similar to that of the known wide companion 
SCR J0753-2524. However, we cannot exclude the possibility that HD $35650 \mathrm{~B}$ is only a chance alignment and represents in fact a distant halo star.

3. Our additional search for nearby WDs using parallaxes from the UPC confirmed our WD selection criteria in two CMDs already applied for our CPM candidates and led to one more WD candidate, UPC 72924 , at $\approx 25 \mathrm{pc}$.

4. Considering the estimated effect pmo of orbital motion on the proper motion difference in CPM pairs, we were able to recover the faint components of known very wide CPM systems (GJ 282AB/GJ 282C and Ross 429/SCR J0753-2524). On the other hand, this allowed us to discover TYC 39801081-1 B as a cool WD companion candidate with relatively small angular separation from a very nearby star, where the pmo effect is very large.

5. HD 166435 B was spectroscopically confirmed as a relatively hot WD, as already suspected from its $G-J=$ -0.54 and large negative GALEX colour index $F U V-N U V$ $\approx-0.67$, whereas TYC 3980-1081-1 B is with $G-J=+1.31$ a cool WD candidate (possibly $\approx$ DA10). Compared to the 25 pc WD sample of Holberg et al. (2016), HD 166435 B ranges as $\approx$ DA2.2 among the hottest nearby DA WDs and is an important addition of a Sirius-like system (a WD with a luminous primary of spectral type K or earlier) in the volume between $20 \mathrm{pc}$ and $25 \mathrm{pc}$.

6. The discovery of TYC 3980-1081-1 B, if confirmed by follow-up spectroscopy, shows that previous assumptions on the completeness of the WD sample within $13 \mathrm{pc}$ may be not correct. This concerns in particular cool WDs with small proper motions.

7. The identification of relatively close companions to very bright stars, such as HD 166435 B and TYC 3980-1081-1 B has always been a challenge with photographic all-sky surveys. We took advantage of the better resolution of CCD images in UCAC5 and Gaia. The next data releases from Gaia will certainly help identify more close WD companions of nearby stars. On the other hand, Limoges et al. (2015) found eight single WDs as probable new members of the $25 \mathrm{pc}$ sample in their photographic (SUPERBLINK) survey of the northern sky with a lower proper motion limit of 40 mas $\mathrm{yr}^{-1}$. A similar number of missing nearby WDs can be expected in the southern sky, and additional WDs with even smaller proper motions and trigonometric distances $<25$ pc, may still be discovered in forthcoming Gaia all-sky catalogues.

We think that the new nearby WD and WD candidates discussed in this paper represent interesting targets for various follow-up observations.

Acknowledgements. We have extensively used the SIMBAD database and the VizieR catalogue access tool operated at the CDS/Strasbourg. The spectroscopic confirmation of one of our WD candidates was part of a larger observing campaign carried out with the $2.2 \mathrm{~m}$ telescope of the German-Spanish Astronomical Centre at Calar Alto, Spain. Part of the observations were carried out in service mode. We would like to thank the Calar Alto staff for their kind support and for their help with the observations. We thank Axel Schwope for providing equivalent widths of Balmer lines in DA WDs. It is a pleasure to thank Benjamin Braun and Peter Grodzewitz for their help with a first look at the TGAS and TGAS/UCAC5 nearby subsamples during their internships at AIP in February and May 2017, respectively. We would like to thank the referee for many valuable comments and suggestions that helped us improve the paper.

\section{References}

Andrews, J. J., Chanamé, J., \& Agüeros, M. A. 2017, MNRAS, 472, 675

Bianchi, L., Herald, J., Efremova, B., et al. 2011, Ap\&SS, 335, 161

Dupuy, T. J., \& Liu, M. C. 2012, ApJS, 201, 19

ESA 1997 The HIPPARCOS and TYCHO catalogues. Astrometric and photometric star catalogues derived from the ESA HIPPARCOS Space Astrometry Mission (Noordwijk, Netherlands: ESA Publications Division), ESA SP, 1200 Finch, C., \& Zacharias, N. 2016a, ArXiv e-prints [arXiv:1604 . 06739] Finch, C. T., \& Zacharias, N. 2016b, AJ, 151, 160

Finch, C. T., \& Zacharias, N. 2016c, VizieR Online Data Catalog: I/333

Finch, C. T., Zacharias, N., Subasavage, J. P., Henry, T. J., \& Riedel, A. R. 2014, AJ, 148, 119

Gaia Collaboration (Brown, A. G. A., et al.) 2016a, A\&A 595, A2

Gaia Collaboration (Prusti, T., et al.) 2016b, A\&A 595, A1

Gaia Collaboration (van Leeuwen, F., et al.) 2017, A\&A, 601, A19

Gaidos, E., Mann, A. W., Lépine, S., et al. 2014, MNRAS, 443, 2561

Gianninas, A., Bergeron, P., \& Ruiz, M. T. 2011, ApJ, 743, 138

Gliese, W., \& Jahreiß, H. 1995, VizieR Online Data Catalog: V/070

Gray, R. O., Corbally, C. J., Garrison, R. F., McFadden, M. T., \& Robinson, P. E. 2003, AJ, 126, 2048

Gray, R. O., Corbally, C. J., Garrison, R. F., et al. 2006, AJ, 132, 161

Henry, T. J., \& Jao, W.-C. 2015, IAU General Assembly, 29, 2253773

Holberg, J. B., Oswalt, T. D., Sion, E. M., \& McCook, G. P. 2016, MNRAS, 462, 2295

Kervella, P., Thévenin, F., \& Lovis, C. 2017, A\&A, 598, L7

Lépine, S. 2011, in 16th Cambridge Workshop on Cool Stars, Stellar Systems, and the Sun, eds. C. Johns-Krull, M. K. Browning, \& A. A. West, ASP Conf. Ser., 448, 1375

Lépine, S. 2017, in AAS Meeting Abstracts, 229, 156.01

Lépine, S., \& Bongiorno, B. 2007, AJ, 133, 889

Lépine, S., \& Shara, M. M. 2005, AJ, 129, 1483

Li, T., Marshall, J. L., Lépine, S., Williams, P., \& Chavez, J. 2014, AJ, 148, 60

Limoges, M.-M., Bergeron, P., \& Lépine, S. 2015, ApJS, 219, 19

Lindegren, L., Lammers, U., Bastian, U., et al. 2016, A\&A, 595, A4

Luyten, W. J. 1995, VizieR Online Data Catalog: I/098

Luyten, W. J. 1997, VizieR Online Data Catalog: I/130

Luyten, W. J. 1998, VizieR Online Data Catalog: I/087

Nelan, E. P., Bond, H. E., \& Schaefer, G. 2015, in 19th Eur. Workshop on White Dwarfs, eds. P. Dufour, P. Bergeron, \& G. Fontaine, ASP Conf. Ser., 493, 501

Oh, S., Price-Whelan, A. M., Hogg, D. W., Morton, T. D., \& Spergel, D. N. 2017, AJ, 153, 257

Poveda, A., Allen, C., Costero, R., Echevarría, J., \& Hernández-Alcántara, A 2009, ApJ, 706, 343

Scholz, R.-D., Meusinger, H., \& Jahreiß, H. 2005, A\&A, 442, 211

Skrutskie, M. F., Cutri, R. M., Stiening, R., et al. 2006, AJ, 131, 1163

Smart, R. L., Marocco, F., Caballero, J. A., et al. 2017, MNRAS, 469, 401

Subasavage, J. P., Henry, T. J., Hambly, N. C., et al. 2005, AJ, 130, 1658

Subasavage, J. P., Jao, W.-C., Henry, T. J., et al. 2009, AJ, 137, 4547

Subasavage, J. P., Jao, W.-C., Henry, T. J., et al. 2017, AJ, 154, 32

Taylor, M. B. 2005, in Astronomical Data Analysis Software and Systems XIV, eds. P. Shopbell, M. Britton, \& R. Ebert, ASP Conf. Ser., 347, 29

Torres, C. A. O., Quast, G. R., da Silva, L., et al. 2006, A\&A, 460, 695

Tremblay, P.-E., Gentile-Fusillo, N., Raddi, R., et al. 2017, MNRAS, 465, 2849

van Leeuwen, F. 2007, A\&A, 474, 653

Zacharias, N., Finch, C., Subasavage, J., et al. 2015, AJ, 150, 101

Zacharias, N., Finch, C., \& Frouard, J. 2017, AJ, 153, 166 